\title{
articles
}

\section{Sleep paralysis and psychopathology}

\author{
Celestine O Mume, BSC, MB ChB, MSc, FMCPsych (Nig) \\ Department of Mental Health, Obafemi Awolowo \\ University, lle-lfe, Nigeria
}

Innocent C Ikem, BM BCh, FMCS (Orthop), FWACS, FICS Department of Orthopaedic Surgery and Traumatology, Obafemi Awolowo University

Background. Sleep paralysis in the absence of narcolepsy is common. Individuals experience episodes of profound muscular paralysis on waking from sleep in the morning or during the night. These episodes are disturbing, especially because they often involve feeling unable to breathe deeply or voluntarily. Previous studies have suggested an association between isolated sleep paralysis (ISP) and adverse psychosocial situations. This study examines an association between ISP and anxiety in orthopaedic patients suffering from physical injuries, patients with multiple somatic complaints (suffering from psychological disorders), and healthy controls.

Methods. Healthy individuals, orthopaedic patients and patients with multiple somatic complaints were asked to fill out a survey that determined the 3-month prevalence of ISP. Anxiety was scored on the Hamilton Anxiety Rating Scale (HARS).

Results. ISP was reported by $28 \%$ of the healthy subjects, $44 \%$ of the orthopaedic patients and $56 \%$ of the patients with multiple somatic complaints. The degree of anxiety among the orthopaedic patients was significantly higher than that in the healthy subjects and significantly lower than that reported by the patients with multiple somatic complaints.

Conclusion. This study suggests that although ISP occurs in healthy individuals, it is more common in association with

Sleep paralysis is a common parasomnia characterised by inability of the individual to move or speak and often accompanied by hallucinations of a sensed presence nearby.' It is one of the less known and more benign forms of parasomnia. The primary or idiopathic form is also called isolated sleep paralysis (ISP). ${ }^{2}$

Hypnagogic and hypnopompic hallucinations are often associated with sleep paralysis. ${ }^{3,4}$ These are visual, somatic, auditory or other hallucinations, usually brief though sometimes prolonged, that occur at the transition from wakefulness to sleep (hypnagogic hallucinations) or from sleep to wakefulness (hypnopompic hallucinations).

Sleep paralysis may occur during the transition from wakefulness to sleep (hypnagogic paralysis) or from sleep to wakefulness (hypnopompic paralysis). It is considered to be a parasomnia related to rapid eye movement (REM) sleep because it tends to occur during awakenings from REM sleep and its pathophysiology is considered to involve muscular atonia mediated through REM sleep. ${ }^{5}$ As with normal physiology during REM sleep, sleep paralysis is characterised by atonia of skeletal muscles. The affected individual is unable to move his or her limbs, head and trunk, although respiration and eye movements remain normal. ${ }^{5}$

Sleep paralysis occurs frequently after arousal from REM sleep, and the hypothesis is that it is caused by cortical awakening before the termination of REM-related atonia. The phenomenon of sleep paralysis represents intrusion of REM sleep atonia into wakefulness. An episode of sleep paralysis usually lasts seconds to minutes, sometimes terminating spontaneously and at other times following an external stimulus such as a sound or when the individual is touched, for example by the bed partner. ${ }^{5}$ Patients characteristically describe these episodes as frightening or even terrifying because they are fully awake and conscious, yet unable to move. Sleep paralysis can occur in isolation or form part of narcolepsy. When it occurs as a component of the narcolepsy syndrome, it tends to occur mostly at initial onset of sleep.

Studies have reported a wide range in the prevalence of ISP..$^{5.7}$ This variation may stem from cultural factors and genetic differences in the populations studied. It may also be due to differences in the methodologies of the studies. It is said that about $30-50 \%$ of healthy individuals experience at least one episode of ISP in their lifetime, ${ }^{5}$ although lower prevalence rates have been reported. In one study ${ }^{7}$ it was found that as few as $5.7-6.7 \%$ of the sample had experienced at least one episode of ISP in their lifetime. In Nigeria it was found that $35.5 \%$ of subjects studied reported having ISP at least twice in the previous year. ${ }^{8}$

Chronic recurrent episodes of sleep paralysis are not common except when they occur in the context of narcolepsy. ${ }^{5}$ An increase in the frequency of sleep paralysis may occur after sleep deprivation and as a result of sleep-wake schedule problems such as work shifts and jet lag syndrome. 
Some authors have reported that recurrent sleep paralysis was more common among African Americans than among whites, especially those suffering from panic disorder. ${ }^{5}$ In one study recurrent sleep paralysis was reported by $59 \%$ of African Americans with panic disorder as opposed to $7 \%$ of whites with the same disorder; it was also reported by $23 \%$ of healthy African American volunteers and $6 \%$ of healthy white volunteers. The higher prevalence among African Americans was attributed to higher levels of psychosocial stressors such as poverty and racism among them, ${ }^{6}$ although it may also partly be due to genetic differences between the races.

Research suggests that rates of ISP are elevated in individuals with post-traumatic stress disorder, panic disorder and other anxiety disorders, ${ }^{6,9-11}$ and in refugees. ${ }^{10}$ It has also been found to be common among psychiatric patients. ${ }^{7}$ Among a psychiatric population of Cambodian refugees, $42 \%$ had had at least one episode of ISP in the previous year. ${ }^{10}$ In an earlier study in Nigeria there was no significant difference in the prevalence of ISP between workers and students. ISP was significantly associated with high scores in the 12-item General Health Questionnaire (GHQ-12) and life events. ${ }^{8}$

The high prevalence of ISP among patients suffering from anxiety disorders and its association with high scores on the GHQ and life events suggest that it is largely associated with psychopathology, although it does occur in healthy individuals.

Individuals who are physically injured tend to develop psychopathology (co-morbidity). This may be through emotional reaction to the physical injuries or through the effects of the physical injuries (or their treatment) on the central nervous system. Patients in an orthopaedic ward who were admitted as a result of physical injuries resulting from combat, road traffic accidents, work accidents, etc. have been found to be at a high risk of psychopathology.

Patients with multiple somatic complaints are another group of individuals suffering from psychological distress. The somatising patient has multiple bodily symptoms, but they are not accounted for by a general medical condition. Even when a general medical condition is present, the symptoms exceed those expected from the general medical condition. Somatisation has been conceptualised as the expression of psychological discomfort and psychosocial distress in the physical language of bodily symptoms.

There are a number of published studies on the association between sleep paralysis and adverse psychosocial situations, $, 0,9-11$ but to our knowledge none so far has investigated the prevalence of sleep paralysis among orthopaedic inpatients and patients with multiple somatic complaints.

\section{Methods}

The study was carried out in the Obafemi Awolowo University Teaching Hospitals Complex (OAUTHC), lle-lfe, south-western Nigeria. The study was approved by the Ethics and Research Committee of the OAUTHC. All the subjects who participated gave written informed consent.

Forty-one patients admitted to the orthopaedic ward (27 males and 14 females) and $25(11$ males and 14 females) who presented with multiple somatic complaints in the psychiatric outpatient clinic were recruited into the study. Fifty healthy individuals 131 males and 19 females) were also recruited into the study, serving as the control group. They included hospital personnel, university personnel, students and business people.

The orthopaedic patients were admitted following physical injuries (such as fracture and dislocation) resulting from combat, road traffic accidents, work accidents and similar circumstances; some of them were recruited postoperatively. Those who were on treatment for co-morbid physical conditions or were too weak to participate were excluded from the study. The psychiatric patients had multiple somatic complaints (such as heat and peppery sensations in the body), had no psychotic symptoms (such as delusions and hallucinations), and had no general medical conditions. The control group consisted of healthy individuals (such as students, teachers and other workers) in lle-lfe.

The subjects were required to complete a questionnaire designed by the authors, and were then rated by one of the authors (COM, who is a psychiatrist) on the Hamilton Anxiety Rating Scale (HARS). ${ }^{12}$ The self-designed questionnaires elicited information on socio-demographic characteristics and the presence or otherwise of at least one episode of ISP within the previous 3 months. The HARS is a clinician-rated standardised instrument widely used to determine the degree of anxiety. The scale consists of 14 items. Each item is scored on a scale of 0 (not present) to 4 (severe), with a total score range of 0 - 56. In this study, anxiety scores on the HARS were used as indices of anxiety among the healthy subjects, orthopaedic patients and patients presenting with multiple somatic complaints. The patients with multiple somatic complaints were clinically diagnosed using the diagnostic criteria of the 10th edition of the International Classification of Diseases (ICD-10). ${ }^{13}$ 
The data were analysed statistically to obtain the sociodemographic characteristics of the subjects in the different groups. The numbers and percentages of those who experienced ISP in the different groups were obtained and compared using the chi-square test. The HARS data were analysed by analysis of variance (ANOVA) (SAS Institute, Cary, NC) and a post hoc test (Student-Newman-Keuls) was carried out to determine the source of a significant effect, significance being taken as $p<0.05$.

\section{Results}

The age range for the subjects in the control group was 18 - 55 years, with a mean age (standard deviation) of 37.4 (9.3) years, while that for the orthopaedic patients was $16-72$ years, with a mean of 38.1 (17.6) years. The age range for the patients with multiple somatic complaints was 17 - 49 years, with a mean of 36. 1 (8.5) years. The socio-demographic characteristics of the different groups of subjects are set out in Table I.

As shown in Fig. 1, 14 of the 50 subjects $(28 \%)$ in the control group and 18 out of the 41 orthopaedic patients (44\%) had ISP. Fifty-six per cent of patients with multiple somatic complaints (14 out of the 25 patients) reported ISP. Chi-square testing showed a significantly ( $p<0.05)$ higher prevalence of ISP among patients with somatic complaints compared with healthy subjects (controls).

As shown in Fig. 2, ANOVA indicated that the mean scores on the HARS for the orthopaedic patients as well as for the patients with multiple somatic complaints were significantly $(F(2,115)=51.63$, $p<0.05)$ higher than the mean score for the control group. The mean score for the patients with multiple somatic complaints was also significantly higher than the score for the orthopaedic patients.

According to the ICD-10,13 the patients who presented with multiple somatic complaints were suffering from anxiety disorders

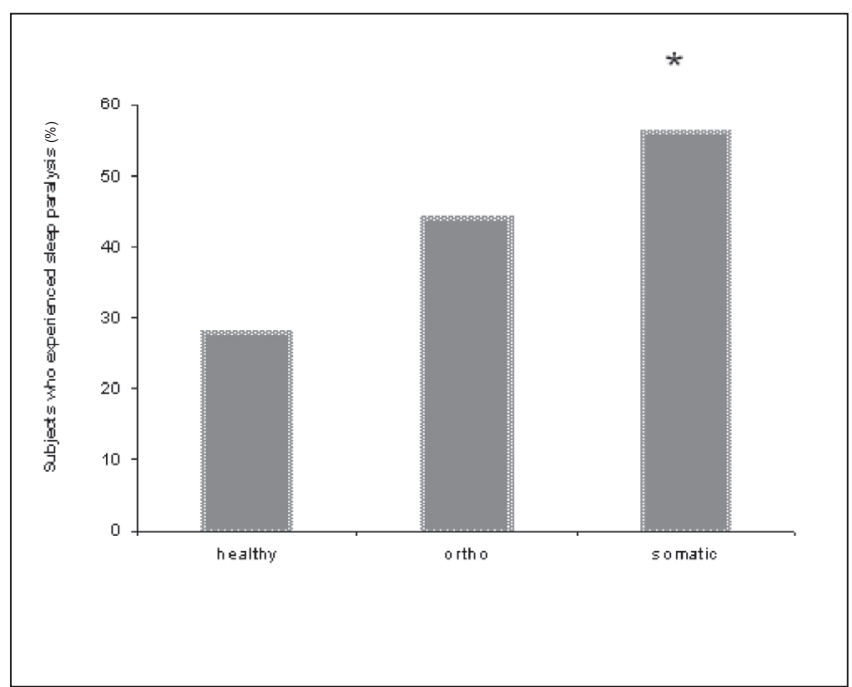

Fig. 1. The bars represent the percentages of healthy subjects (healthy, $\mathrm{N}=50$ ), orthopaedic patients (ortho, $\mathrm{N}=41$ ) and patients with multiple somatic complaints (somatic, $\mathrm{N}=25$ ) who reported ISP ( ${ }^{*} \mathrm{p}<0.05 \mathrm{v}$. healthy subjects (controls)). Twentyeight per cent of healthy subjects, $44 \%$ of orthopaedic patients and $56 \%$ of patients with multiple somatic complaints reported ISP. Chi-square testing showed that ISP was significantly commoner among patients with multiple somatic complaints compared with the controls.

$160 \%$ ), a depressive episode (24\%) and somatisation disorder $(16 \%)$.

\section{Discussion}

Sleep paralysis characteristically occurs as the subject wakes from REM sleep before muscular paralysis wanes. Individuals describe the experience of not being able to move and being pressed down. It is also often accompanied by hallucinations of a sensed presence, often of threatening intruders in the bedroom.

There is a traditional view that sleep paralysis reflects the intrusion of some enemies, evil spirits and supernatural forces into sleeping

Table I. Socio-demographic characteristics of the subjects in the different groups

\begin{tabular}{|c|c|c|c|c|}
\hline Variable & & Healthy subjects & Orthopaedic patients & $\begin{array}{l}\text { Patients with multiple somatic } \\
\text { complaints }\end{array}$ \\
\hline Age range (yrs) & & $18-55$ & $16-72$ & $17-49$ \\
\hline \multirow[t]{2}{*}{ Gender } & Male & 31 & 27 & 11 \\
\hline & Female & 19 & 14 & 14 \\
\hline \multirow[t]{5}{*}{ Marital status } & Single & 17 & 14 & 10 \\
\hline & Married & 29 & 27 & 9 \\
\hline & Separated & 3 & 0 & 2 \\
\hline & Divorced & 1 & 0 & 3 \\
\hline & Widowed & 0 & 0 & 1 \\
\hline
\end{tabular}




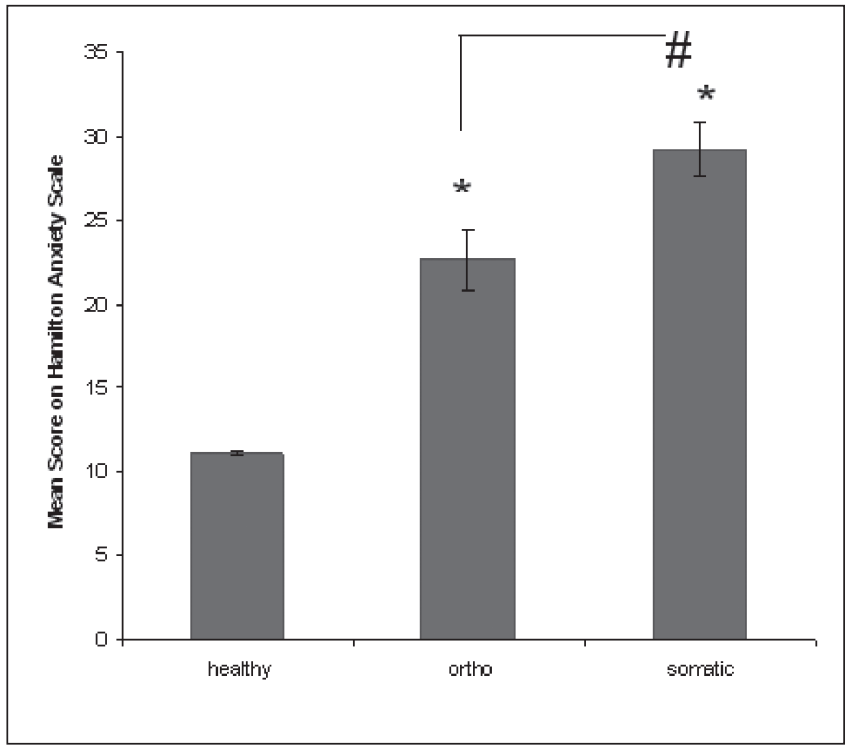

Fig. 2. HAS scores (mean (standard error)) for the healthy subjects (healthy, $\mathrm{N}=50$ ), orthopaedic patients (ortho, $\mathrm{N}=41$ ) and patients with multiple somatic complaints (somatic, $\mathrm{N}=25$ ) $\left({ }^{*} \mathrm{p}<0.05 \mathrm{v}\right.$. healthy subjects (controls), \#p $<0.05 \mathrm{v}$. orthopaedic patients). ANOVA showed that the mean HAS scores for the orthopaedic patients as well as for the patients with multiple somatic complaints were significantly higher than the mean score for the controls.

people. ${ }^{14}$ It is therefore a source of considerable distress to the sufferers, their bed partners and other family members.

The increased prevalence of ISP observed in the different groups in this study /lowest in the healthy subjects, intermediate in the orthopaedic patients and highest in patients presenting with somatic complaints) is consistent with the degree of anxiety observed in the groups as shown by the scores on the HARS. The study indicates that the higher the degree of anxiety, the higher the prevalence of sleep paralysis.

It is sometimes difficult to compare prevalence rates in different studies owing to differences in sample size as well as differences in the period of time for which the rate is calculated. However, the 3-month prevalence of $28 \%$ among healthy subjects reported in this study appears consistent with previous findings. In one of the earlier studies on ISP done in the general population in
Nigeria, $18.2 \%$ of the subjects experienced it once in a month, ${ }^{15}$ and a 1 -year prevalence rate of $35.5 \%$ was reported. ${ }^{8}$ Among a psychiatric population of Cambodian refugees $42 \%$ had experienced sleep paralysis at least once in the previous year. ${ }^{10}$

This study has shown that ISP is common among the groups studied. The association between ISP and anxiety suggests that ISP poses a public health concern. Similarly, a previous study suggested that ISP was often associated with a mental disorder.'

A limitation of this study was the small sample size. Further studies are required to determine the nature of the association between sleep paralysis and anxiety. It is important to determine whether sleep paralysis precipitates mental disorder or whether mental disorder increases the risk of developing sleep paralysis.

\section{References}

1. Solomonova E, Nielsen T, Stenstrom P, Simard V, Frantova E, Donderi D. Sensed presence as a correlate of sleep paralysis distress, social anxiety and waking state social imagery. Conscious Cogn 2008; 17: 49-63

2. de Jong JT. Cultural variation in the clinical presentation of sleep paralysis. Transcult Psychiatry 2005; 42: 78-92

3. Kompanje EJ. 'The devil lay upon her and held her down.' Hypnagogic hallucinations and sleep paralysis described by the Dutch physician Isbrand van Diemerbroeck (1609-1674) in 1664. J Sleep Res 2008; 17: 464-467

4. Cheyne JA. Sleep paralysis episode frequency and number, types, and structure of associated hallucinations. J Sleep Res 2005; 14: 319-324

5. Peretz L, Giora P, Atul M. Sleep Disorders. 1st ed. London: Martin Dunitz, 2002: 156-158

6. Paradis CM. Friedman S. Sleep paralysis in African Americans with panic disorder. Transcult Psychiatry 2005; 42: 123-134

7. Ohayon MM, Zulley J, Guilleminault C, Smirne S. Prevalence and parhologic associations of sleep paralysis in the general population. Neurology 1999; 52 1 194-1200.

8. Ohaeri JU. The prevalence of isolated sleep paralysis among a sample of Nigerian civil servants and undergraduates. Afr J Med Med Sci 1997; 26: 43-45.

9. Otto MW, Simon NM, Powers M, Hinton D, Zalta AK, Pollack MH. Rates of isolated sleep paralysis in outpatients with anxiety disorders. J Anxiety Disord 2006; 20: 687693.

10. Hinton DE, Pich V, Chhean D, Pollack MH, McNally RJ. Sleep paralysis among Cambodian refugees: association with PTSD diagnosis and severity. Depress Anxiety 2005; 22: 47-51

1 1. Yeung $A, X \cup Y, C$ Chang DF. Prevalence and illness beliefs of sleep paralysis among Chinese psychiatric patients in China and the United States. Transcult Psychiatry 2005; 42: 135-145

12. Hamilton M. The assessment of anxiety states by rating. Br J Med Psychol 1959; 32 50-55

13. World Health Organization. The ICD-10 Classification of Mental and Behavioural Disorders. Geneva: WHO, 1992

14. Law S, Kirmayer LJ. Invit interpretations of sleep paralysis. Transcult Psychiatry 2005 42: 93-1 12

15. Ohaeri JU, Awadalla A, Makanjuola VA, Ohaeri BM. Features of isolated sleep paralysis among Nigerians. East Afr Med J 2004; 81 : 509-519. 